\title{
Proposta Metodológica para a Avaliação da Técnica da Pedalada de Ciclistas: Estudo de Caso
}

\section{Methodological Proposal for Evaluation of the Pedaling Technique of Cyclists: a Case Study}

\author{
Fernando Diefenthaeler \\ Rodrigo Rico Bini ${ }^{1}$ \\ Eduardo Nabinger ${ }^{2}$ \\ Orlando Laitano ${ }^{1}$ \\ Felipe Pivetta Carpes ${ }^{2}$ \\ Carlos Bolli Mota ${ }^{3}$ \\ Antônio Carlos Stringhini Guimarães \\ (in memoriam)
}

1. UFRGS/Laboratório de Pesquisa do Exercício, Porto Alegre - RS; 2. UFRGS/Laboratório de Medições Mecânicas, Porto Alegre - RS; 3. UFSM/Laboratório de Biomecânica, Santa Maria - RS.

\section{Endereço para correspondência: Laboratório de Pesquisa do Exercício, Escola da Educação Física, Universidade Federal do Rio Grande do Sul. \\ Rua Felizardo 750, Lapex, sala 212. \\ Telefone: 3316-5859.}

Email: fdiefenthaeler@gmail.com

Submetido em 14/08/2006

Versão final recebida em 05/11/2007

Aceito em 09/12/2007

\begin{abstract}
RESUMO
No estudo da biomecânica do ciclismo diversas técnicas têm sido utilizadas para descrever e compreender o movimento da pedalada. O objetivo deste estudo é propor uma metodologia para a avaliação de ciclistas sob o ponto de vista das forças aplicadas no pedal. Um ciclista de elite foi avaliado por meio de um protocolo que consistiu em alterar o selim em quatro diferentes posições (deslocado para cima, para baixo, para frente e para trás) a partir da posição de referência, especificamente, aquela adotada para treinamento pelo ciclista. A mudança no ajuste do selim foi de $1 \mathrm{~cm}$. A bicicleta do atleta foi acoplada a um ciclossimulador magnético. A carga do teste foi normalizada por um critério fisiológico (segundo limiar ventilatório), simulando o ritmo de prova do atleta. O pedal direito foi substituído por um pedal bidimensional instrumentado para registrar as forças normal e tangencial. A média do impulso angular da força efetiva foi calculada a partir de dez ciclos consecutivos de pedalada. As mudanças na posição do selim modificaram o impulso da força efetiva em relação à posição de referência. Sendo assim, o protocolo é eficaz e pode ser aplicado em diferentes situações.
\end{abstract}

Palavras-chave: ciclismo, biomecânica, desempenho.

\begin{abstract}
Many techniques have been used in biomechanics to describe the cycling movement. The purpose of this study is to proposal a specific methodology to evaluation the forces applied on the pedal. An experienced elite cyclist was submitted to a protocol which consisted of four different saddle positions (upward, downward, forward, and backward) assuming as reference position the one used by the cyclist in training and competition. The displacement of the saddle was of $1 \mathrm{~cm}$ in all tests. The individual's bicycle was connected to a magnetic cycle simulator. The load was normalized by a physiological criterion (ventilatory threshold), to simulate the cyclist's race rhythm. The right regular pedal was replaced by a 2D instrumented pedal to record the force normal and tangential components applied on it. Mean impulse of the angular effective force was calculated from ten consecutive pedaling cycles. The four different saddle positions have modified the effective angular pedaling impulse comparing with the reference position. Relatively small saddle adjustments may affect the effective angular pedaling impulse and supposedly cycling performance.
\end{abstract}

Keywords: cycling, biomechanics, performance.

\section{INTRODUÇÃO}

Para estudar a biomecânica do ciclismo diversas ferramentas têm sido utilizadas para a melhor compreensão do movimento da pedalada. A grande variedade de procedimentos adotados em estudos envolvendo ciclismo tem levado os pesquisadores a discutir a aplicação dos mesmos ${ }^{(1-3)}$. Dentre os protocolos utilizados em avaliações de ciclistas, destaca-se a utilização simultânea de parâmetros fisiológicos e biomecânicos na tentativa de elucidar as variáveis relacionadas ao gesto da pedalada ${ }^{(3-8)}$.

Em avaliações fisiológicas de ciclistas de alto rendimento normalmente tem sido utilizado o consumo máximo de oxigênio $\left(\mathrm{VO}_{2 \mathrm{MÁx}}\right)$, a concentração de lactato sangüíneo, os limiares ventilatórios, a economia de movimento (razão entre o trabalho realizado e a energia despendida) e a relação do tipo de fibra muscular como indicadores da capacidade aeróbia ${ }^{(8-10)}$. Já nas avaliações biomecânicas do ciclismo, além do uso das técnicas de cinemetria e de eletromigrafia, a utilização de pedais instrumentados tem permitido mensurar as forças aplicadas no pedal, assim como entender como estas são aproveitadas ao longo do ciclo, determinando desta forma a técnica da pedalada(5,11-15).

A maior parte dos estudos encontrados na literatura, envolvendo a avaliação da técnica da pedalada, utilizaram cargas fixas e iguais para todos os sujeitos, com valores variando entre 100 e $250 W^{(11,13,16-18)}$. Entretanto, na maioria dos casos, estas cargas subestimam a intensidade do esforço de ciclistas de elite durante os treinamentos e, principalmente, as competições.

Desta forma, a aplicação de um protocolo utilizando um critério fisiológico como normalização da carga na avaliação da técnica da pedalada de ciclistas de elite tem sido proposto na literatura, permitindo que a carga do teste seja muito próxima à intensidade de competição $0^{(4,8,19)}$. Essa metodologia permite um aprimoramento dos trabalhos que fixaram uma mesma carga para todos os sujeitos ${ }^{(1,13,16-18)}$, pois respeita as características fisiológicas individuais.

Mesmo que a normalização fisiológica gere respostas relevantes 
do ponto de vista biomecânico (magnitude e direcionamento das forças aplicadas no pedal), os protocolos supracitados utilizam bicicletas ergométricas que apresentam limitações para os diferentes ajustes necessários (i.e. altura do selim, tamanho do pé-de-vela, ângulo entre os tubos, entre outras medidas), para cada indivíduo avaliado.

Sendo assim, o objetivo deste trabalho é propor uma metodologia para a avaliação de ciclistas utilizando um critério fisiológico como normalização da carga e a própria bicicleta do ciclista, a qual ele está adaptado, diferentemente dos estudos citados anteriormente, que utilizaram cicloergômetro. Portanto, trabalhar essa questão de forma individualizada torna-se essencial em se tratando de atletas de alto nível do ciclismo, do triatlo, do mountain bike ou de qualquer outra modalidade envolvendo bicicleta. Neste estudo, a metodologia proposta foi empregada a fim de investigar as forças aplicadas no pedal em diferentes ajustes do selim.

\section{MATERIAIS E MÉTODOS}

Para este estudo foi avaliado um ciclista de estrada da elite do estado do Rio Grande do Sul, Brasil. O atleta concordou em participar do estudo de forma voluntária e assinou o termo de consentimento informado. Este trabalho foi aprovado pelo Comitê de Ética em Pesquisa da Universidade Federal do Rio Grande do Sul, Brasil. O ciclista foi avaliado em dois dias, com intervalo de 24 horas.

Primeiro dia de avaliação: Anteriormente ao início do teste, foram realizadas avaliações cineantropométricas para caracterização do sujeito: (a) massa corporal e estatura; (b) perímetros corporais; e (c) dobras cutâneas. Estas medidas foram utilizadas com base no protocolo proposto na literatura ${ }^{(20,21)}$. Os resultados desta avaliação são apresentados na Tabela 1.

Tabela 1. Características individuais e antropométricas do ciclista avaliado.

\begin{tabular}{c|c|c|c|c|c}
\hline $\begin{array}{c}\text { Idade } \\
\text { (anos) }\end{array}$ & $\begin{array}{c}\text { Massa } \\
\text { corporal (kg) }\end{array}$ & $\begin{array}{c}\text { Estatura } \\
(\mathbf{m})\end{array}$ & $\begin{array}{c}\text { Percentual de } \\
\text { gordura (\%) }\end{array}$ & $\begin{array}{c}\text { Tempo de } \\
\text { treino (anos) }\end{array}$ & $\begin{array}{c}\text { Volume } \\
\text { semanal de } \\
\text { treino (km) }\end{array}$ \\
\hline 25 & 63,3 & 1,66 & 7,9 & 8 & 450 \\
\hline
\end{tabular}

Após esta etapa foi avaliado o $\mathrm{VO}_{2 \mathrm{MAX}}$ do ciclista usando um sistema de ergoespirometria de circuito aberto por analisador de gases modelo CPX/D (Medical Graphics Corp., St Louis, EUA) em um cicloergômetro

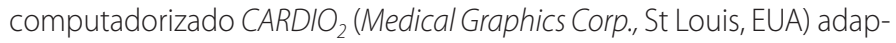
tado com selim e pedais de bicicletas de competição, permitindo que o ciclista utilizasse sua própria sapatilha. Foi utilizado protocolo de rampa com incremento de carga de $30 \mathrm{~W} \cdot \mathrm{min}^{-1}$ até a exaustão (ou interrompido quando o atleta não conseguisse manter a cadência acima de 70 rpm) para a determinação do consumo de oxigênio $\left(\mathrm{VO}_{2}\right)$, segundo limiar ventilatório $\left(\mathrm{LV}_{2}\right)$ e taxa de troca respiratória (RER).

A verificação do $\mathrm{VO}_{2}$ e do RER correspondente ao $\mathrm{LV}_{2}$ permitiu a determinação da carga de trabalho para o segundo dia de avaliação( ${ }^{(8)}$. A freqüência cardíaca (FC) foi monitorada por meio de um freqüêncímetro Polar S610 (Polar Electro Oy, Finlândia) durante todo o teste máximo.

Na Tabela 2 estão detalhadas as características fisiológicas do atleta avaliado obtidas durante o teste máximo realizado no primeiro dia de testes.

Tabela 2. Características fisiológicas do ciclista avaliado.

\begin{tabular}{c|c|c|c|c|c}
\hline $\begin{array}{c}\mathrm{VO}_{2 \text { MAX }} \\
\left(\mathbf{m l} \cdot \mathbf{k g}^{-1} \cdot \mathbf{m i n}^{-1}\right)\end{array}$ & $\begin{array}{c}\text { RER no } \\
\mathrm{VO}_{2 \text { MAX }}\end{array}$ & $\begin{array}{c}\mathrm{FC}_{\text {MAX }} \\
(\mathbf{b p m})\end{array}$ & $\begin{array}{c}\mathrm{VO}_{2} \text { no LV } \\
\left(\mathbf{m l} \cdot \mathbf{k g}^{-1} \cdot \mathbf{m i n}^{-1}\right)\end{array}$ & $\begin{array}{c}\text { RER no } \\
\mathrm{LV}_{2}\end{array}$ & $\begin{array}{c}\mathrm{FC} \text { no } \mathrm{LV}_{2} \\
(\mathbf{b p m})\end{array}$ \\
\hline 76,7 & 1,18 & 192 & 63 & 1,01 & 177 \\
\hline
\end{tabular}

Segundo dia de avaliação: o ciclista foi avaliado utilizando a sua própria bicicleta acoplada a um ciclossimulador magnético Cateye CS1000 (Cateye CO. Osaka, Japão) com ajuste de carga em watts e inclinação em graus. O ciclossimulador foi ajustado de modo a simular um aclive de 3\%. Antes do início do teste, o ciclista teve um período de 5 minutos para aquecimento e para o ajuste da relação de marchas e da cadência (preferida pelo atleta), que foram, então, mantidas constantes durante todo o protocolo.

O selim da bicicleta foi ajustado em quatro diferentes posições a partir da posição de referência (selim na posição adotada usualmente em treinamento e competições) durante a avaliação: (a) deslocado para cima; (b) deslocado para baixo; (c) deslocado para frente; e (d) deslocado para trás. O deslocamento do selim em todas as posições foi de $1 \mathrm{~cm}$.

Para a troca da posição do selim foi definido um período de repouso de 30 segundos. Um dos avaliadores realizava a mudança do selim para a posição previamente demarcada no canote do selim da bicicleta. Durante este período o atleta permanecia de pé na bicicleta, apoiando-se exclusivamente nos pedais e no guidão.

Ao longo do teste o $\mathrm{VO}_{2}$ e o RER foram mantidos em um valor correspondente ao $\mathrm{LV}_{2}$ com o objetivo de normalizar a carga e simular uma intensidade muito próxima do seu ritmo de competição. $\mathrm{O} \mathrm{VO}_{2}$ permaneceu constante durante as cinco posições avaliadas, aceitandose uma variação de $\pm 1,8 \mathrm{ml} \cdot \mathrm{kg}^{-1} \cdot \mathrm{min}^{-1}$ entre as posições testadas. Este valor é recomendado pelo American College of Sports Medicine ${ }^{(22)}$, uma vez que esta mudança não representa uma alteração importante no custo metabólico. O RER determina a predominância de qual sistema energético está sendo utilizado pelo atleta, sendo que valores abaixo de 1 indicam uma maior contribuição do sistema aeróbio e valores acima de 1 indicam maior contribuição do sistema anaeróbio(23,24). 0 RER manteve-se entre 0,9 e 1 indicando que o atleta pedalou em uma intensidade muito próxima ao $\mathrm{LV}_{2}^{(2,25,26)}$.

Sob as condições descritas o atleta pedalou com o selim ajustado em cada uma das cinco posições até atingir e estabilizar, por três minutos, o VO, e o RER. Nos 30 segundos finais foram coletados simultaneamente os sinais de dinamometria (forças aplicadas no pedal), eletrogoniometria (ângulos referentes ao movimento do pedal e do pé-de-vela), variáveis fisiológicas $\left(\mathrm{VO}_{2}, \mathrm{RER}\right.$ e $\left.\mathrm{FC}\right)$, velocidade $\left(\mathrm{km} \cdot \mathrm{h}^{-1}\right)$ e cadência (RPM) de pedalada.

Para a medição das componentes de força aplicadas no pedal foi utilizado um pedal-plataforma bidimensional(27) acoplado no pé-devela (PDV) direito. Para o registro do ângulo do pé-de-vela, foi utilizado um sensor eletromagnético, do tipo reed switch, fixado no quadro da bicicleta, próximo ao movimento central, e um imã fixado ao pé-de-vela esquerdo. A posição do sensor em relação ao ponto morto superior do PDV (0॰) foi medida com o auxílio de um goniômetro manual. 0 sensor permitiu a determinação do início e do fim de cada ciclo da pedalada ${ }^{(28-32)}$, conforme ilustra a Figura 1. Para o registro do ângulo do pedal relativo ao pé-de-vela, foi utilizado um sensor angular (potenciômetro) acoplado ao eixo do pedal direito ${ }^{(33)}$.

Todos os sinais foram convertidos por uma placa analógico-digital de 16 canais CODAS (Data Instruments, Inc. Akron, EUA) com resolução de 16 bits. Os dados foram adquiridos pelo software CODAS e armazenados em um microcomputador modelo PC Pentium $200 \mathrm{MHz}$. A freqüência de amostragem dos sinais de dinamometria e eletrogoniometria foi de $2300 \mathrm{~Hz}$ por canal. O processamento dos sinais foi realizado utilizando-se rotinas implementadas no software SAD (Laboratório de Medições Mecânicas, Escola de Engenharia, UFRGS, www.ufrgs.br/lmm).

Para os sinais de força e de eletrogoniometria foi utilizado um filtro digital do tipo ideal passa baixa com freqüência de corte de $10 \mathrm{~Hz}$. Os sinais de dinamometria e de eletrogoniometria foram normalizados na escala 


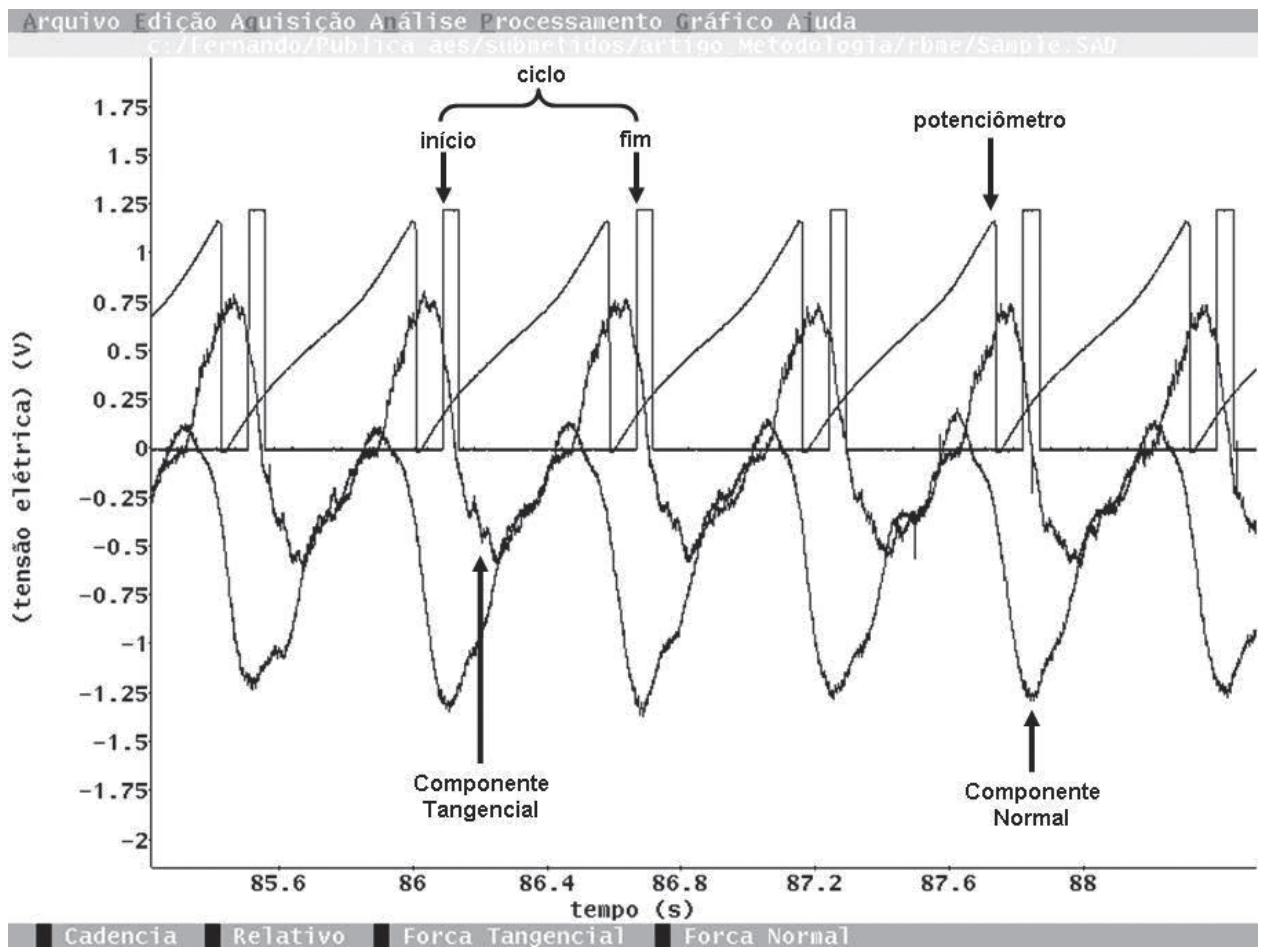

Figura 1. Os sinais brutos coletados durante a avaliação. No eixo vertical os valores são expressos em volts (V) e no eixo horizontal em tempo (s). As setas indicam o início e o fim de cada ciclo de pedalada, registrado com o auxílio de um sensor reed switch fixado no quadro da bicicleta. O sinal do potenciômetro fornece o ângulo relativo entre o pedal e o PDV. As componentes de força normal e tangencial são registradas ao longo do ciclo de pedalada.
Observa-se que o ciclista avaliado apresentou um maior $\mathrm{IF}_{\mathrm{E}}$ na posição de referência. Lembrando que o $\mathrm{IF}_{\mathrm{E}}$ é a diferença entre a força efetiva positiva (propulsiva) e a força efetiva negativa (resistiva) aplicada no pé-de-vela ao longo do ciclo(13). Isto justifica o uso do impulso como um meio de quantificar o desempenho de um ciclista em diferentes situações (i.e. ajustes na posição do selim, ajustes na altura do guidão e diferentes angulações de quadro), visto que este representa o quanto da força aplicada no pé-de-vela gera propulsão na bicicleta.

Os resultados deste estudo apresentam valores distintos de $\mathrm{IF}_{\mathrm{E}}$ nas cinco posições avaliadas. Tal fato possivelmente ocorre devido à adaptação funcional e a propriedade mecânica muscular força-comprimento. A mudança nas relações força-comprimento e força-velocidade provocam alterações na capacidade de produção de força e, conseqüentemente, na produção de potência(35,36). de tempo em relação à curva que possuía o menor número de pontos (menor cadência) para a realização da média. Para todas as variáveis foi calculada a média dos dez primeiros ciclos alternados de pedalada.

Esta normalização pôde ser feita visto que a cadência do atleta manteve-se constante ao longo dos testes, por se tratar de um indivíduo altamente treinado. Isto foi testado por meio da análise do coeficiente de variação da cadência média nos dez ciclos (1,21\% ou 1,21 RPM como maior variação na posição com o selim mais para cima).

Com o objetivo de facilitar a análise e a discussão dos resultados, dividiu-se o ciclo da pedalada em fase de propulsão $\left(0-180^{\circ}\right)$ e fase de recuperação $\left(180-360^{\circ}\right)$, conforme proposto na literatura ${ }^{(34)}$.

Para o cálculo da força efetiva $\left(F_{E}\right)$, que consiste na componente de força aplicada perpendicular ao pé-de-vela gerando propulsão, foi realizada a decomposição das componentes da força normal $\left(F_{y}\right)$ e da força tangencial $\left(F_{x}\right)$ em relação ao pé-de-vela(33).

Para analisar a força efetiva, expressa em função do ângulo do péde-vela, convencionou-se que os valores positivos representam a força que é propulsiva, isto é, que gera torque a favor do movimento do PDV (sentido horário). Os valores negativos representam a força resistiva, ou seja, a força que gera torque no sentido oposto ao movimento do PDV (sentido anti-horário).

Foi calculada também a integral da curva de força efetiva em cada posição avaliada para a determinação do impulso gerado por esta, como mostra a Figura 2. Para a análise dos resultados, o impulso da força efetiva, em cada posição do selim, foi normalizado pelo valor da posição de referência. Não foram utilizados procedimentos estatísticos, uma vez que este trabalho apresenta-se na forma de um estudo de caso.

\section{RESULTADOS E DISCUSSÃO}

Os resultados do impulso da força efetiva $\left(I_{E}\right)$ nas cinco posições avaliadas são apresentados na Tabela 3, e estão expressos em valores absolutos e percentuais.

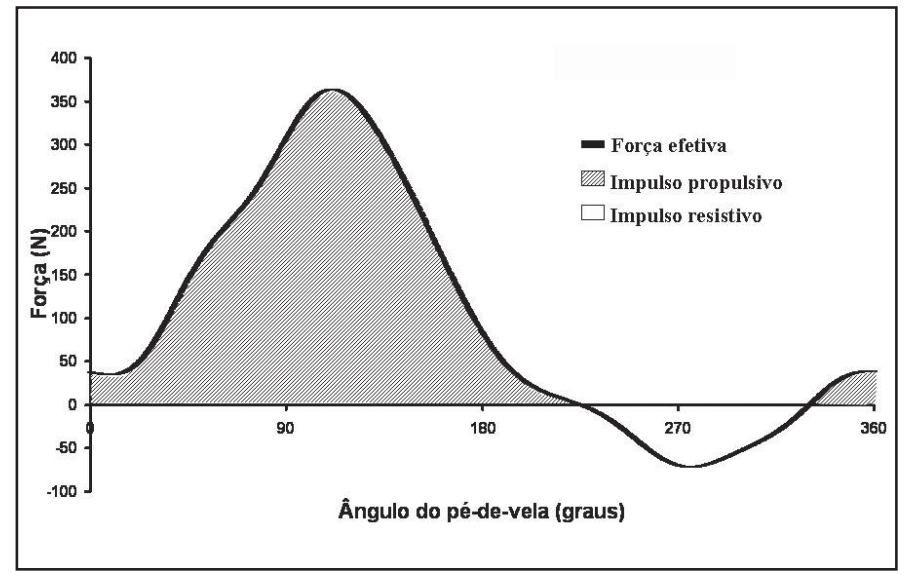

Figura 2. Gráficos da média de 10 ciclos de pedalada do ciclista avaliado, com o selim na posição de referência: força efetiva; impulso propulsivo e impulso resistivo, expressos em função do ângulo do pé-de-vela.

Tabela 3. Impulso angular médio e normalizado (pela posição de referência) da força efetiva nas cinco posições do selim avaliadas.

\begin{tabular}{c|c|c|c|c|c}
\hline Posição do selim & referência & cima & baixo & frente & trás \\
\hline Impulso (N.s) & 55,97 & 52,05 & 53,73 & 50,93 & 52,61 \\
\hline Impulso (\%) & 100 & 93 & 96 & 91 & 94 \\
\hline
\end{tabular}

Partindo do pressuposto de que a velocidade de pedalada (cadência) manteve-se constante ao longo dos testes, assume-se que as alterações na magnitude e na direção das forças, nas distintas posições, estão relacionadas com mudanças na propriedade força-comprimento dos músculos envolvidos no movimento. As mudanças na posição do selim, alterando a relação força-comprimento, possivelmente, interfe- 
rem na técnica da pedalada, o que é identificado pelas alterações na aplicação das forças no pedal ${ }^{(1)}$.

A mudança na posição do membro inferior, em relação ao pedal, possivelmente modifica o direcionamento da força aplicada no pé-devela alterando, por sua vez, o aproveitamento desta. O direcionamento das componentes normal $\left(F_{Y}\right)$ e tangencial $\left(F_{X}\right)$ determina a habilidade técnica do ciclista em transmitir a força total aplicada no pedal para o pé-de-vela ${ }^{(37,38)}$.

Dessa forma, à medida que o ciclista adquire a habilidade de gerar torque propulsor durante a fase de recuperação, ocorre redução do torque resistivo produzido pelo peso do próprio segmento e, conseqüentemente, aumento no impulso da força efetiva( ${ }^{(39,40)}$.

Neste estudo, foram apresentados somente os resultados do $\mathrm{IF}_{\mathrm{E}}$ como forma de aplicação da metodologia proposta para avaliação de ciclistas. Cabe ressaltar, que o impulso da força efetiva tem sido proposto como o melhor parâmetro para representar o aproveitamento das forças aplicadas nos pedais, bem como um excelente indicador da técnica de pedalada $(8,33,39,40)$.

Seria possível ainda analisar as forças aplicadas no pedal isoladamente (magnitude e direcionamento); a força resultante (somatório das componentes de força normal e tangengial aplicadas no pedal) bem como o impulso da força resultante; e a relação entre a força efetiva e a força resultante, chamado de índice de efetividade.

No entanto, é importante destacar que o objetivo deste estudo foi propor um critério de avaliação simples e eficaz, utilizando a própria bicicleta do atleta, em uma intensidade que seja capaz de fornecer informações pertinentes a ciclistas e técnicos a fim de melhorar o desempenho em competições.

\section{CONCLUSÕES}

A metodologia de avaliação proposta neste estudo mostrou-se eficaz, aproximando-se à realidade de treinamento e competição do atleta. O protocolo pode ser realizado periodicamente e/ou em momentos específicos do treinamento de ciclistas e também de triatletas, podendo ser adequado a diferentes situações além das testadas neste estudo, como por exemplo, para testes de diferentes tamanhos de pé-de-vela ou tipos de guidom.

Como direção futura seria importante frisar o uso de outras técnicas utilizadas em avaliações biomecânicas (cinemetria e eletromiografia) para melhor compreender a aplicação das forças, bem como o uso de dois pedais instrumentados, o que tornaria a avaliação mais precisa, principalmente se o objetivo for analisar a assimetria em determinadas situações.

\section{AGRADECIMENTOS}

Os autores gostariam de agradecer ao CAPES e ao CNPq pelo apoio financeiro, a Cateye $\mathrm{CO}$, pela doação do ciclossimulador usado nesse estudo, e ao eterno mestre e amigo Prof. Antônio Carlos Stringhini Guimarães.

Todos os autores declararam não haver qualquer potencial conflito de interesses referente a este artigo.

\section{REFERÊNCIAS BIBLIOGRÁFICAS}

1. Too D. Biomechanics of cycling and factors affecting performance. Sports Med 1990; 10: 286-303.

2. Moseley L, Jeukendrup AE. The reliability of cycling efficiency. Med Sci Sports Exerc 2001; 33: 621-7.

3. Lucia A, Hoyos J, Pérez M, Santalla A, Chicharro JL. Inverse relationship between $\mathrm{VO}_{2 \text { MAX }}$ and economy/ efficiency in world-class cyclists. Med Sci Sports Exerc 2002; 34: 2079-84.

4. Coyle EF, Feltner ME, Kautz SA, Hamilton MT, Montain SJ, Baylor AM, et al. Physiological and biomechanical factors associated with elite endurance cycling performance. Med Sci Sports Exerc 1991; 23: 93-107.

5. Álvarez G, Vinyolas J. A new bicycle pedal design for on-road measurements of cycling forces. J Appl Biomech 1996; 12: 130-42.

6. Caldwell GE, McCole SD, Hagberg JM. Pedal and crank kinetics in uphill cycling. J Appl Biomech $1998 ; 14: 245-59$.

7. Pringle JSM, Jones AM. Maximal lactate steady state, critical power and EMG during cycling. Eur J Appl Physiol 2002; 88: 214-26.

8. Candotti CT, Ribeiro J, Soares DP, Oliveira AR, Loss JF, Guimarães ACS. Effective force and economy of triathletes and cyclists. Sport Biomech 2007; 6: 32-43.

9. Coyle EF, Sidossis LS, Horowitz JF, Beltz JD. Cycling efficiency is related to the percent of type I muscle fiber. Med Sci Sports Exerc 1992; 24: 782-8.

10. Ribeiro JP, Yang J, Adams RP, Kuca B, Knutten HG. Effect of different incremental exercise protocols on the determination of lactate and ventilatory thresholds. Braz J Med Biol Research 1986; 19: 109-17.

11. Hull ML, Davis RR. Measurement of pedal loading in bicycling: I. Instrumentation. J Biomech $1981 ; 14: 843-56$.

12. LaFortune MA, Cavangh PR. Effectiveness and efficiency during bicycle riding. In: Matsui, Kobashi K (ed). Biomechanics VIII-B. Champaign: Human Kinetics 1983; 928-36.

13. Sanderson DJ. The influence of cadence and power output on the biomechanics of force application during steady-state cycling in competitive and recreational cyclists. J Sports Sc 1991; 9: 191-3.

14. Broker JP, Gregor RJ, Schimidt RA. Extrinsic feedback and learning of kinetics patterns in cycling. J Appl Biomech 1993; 9: 111-23.

15. Nabinger E. Desenvolvimento de um sistema de medição para análise biomecânica da pedalada de um ciclista. Dissertação de mestrado. PPGCMH/UFRGS 1997.

16. Davis RR, Hull ML. Measurement of pedal loading in bicycling: II. Analysis and results. J Biomech 1981; 14: 857-72.

17. Ericson MO, Nisell R. Efficiency of pedal forces during ergometer cycling. Int J Sports Med 1988; 9: 118-22

18. Marsh AP, Martin PE, Sanderson DJ. Is a joint moment-based cost function associated with preferred cycling cadence? J Biomech 2000; 33: 173-80.

19. Cannon DT, Kolkhorst FW, Cipriani DJ. Effect of pedaling technique on muscle activity and cycling efficiency. Eur J Appl Physiol 2007; in press.

20. Hawes MR. Human body composition. In: Eston R, Reilly T (ed). Kineanthropometry and exercise physiology laboratory manual. London: E \& FN Spon 1996; 5-31
21. Jackson AS, Pollock ML. Generalized equations for predicting body density of men. Br J Nutr 1978; 40: 497-04

22. American College of Sports Medicine. Diretrizes do ACSM para os testes de esforço e sua prescrição. Sexta edição. Rio de Janeiro: Guanabara Koogan 2003.

23. Frayn KN. Calculation of substrate oxidation rates in vivo from gaseous exchange. J Appl Physiol 1983; 16: 628-34

24. Péronnet F, Massicotted D. Table of Nonprotein respiratory quotient: an update. Can J Sport Sci 1991; 23-9.

25. McLellan TM, Gass GC. Metabolic and cardiorespiratory responses relative to the anaerobic threshold. Med Sci Sports Exerc 1989; 21: 191-8.

26. Figueira TR, Denadai BS. Relações entre limiar anaeróbio, limiar anaeróbio individual e máxima fase estável de lactato em ciclistas. Rev Bras Cien Mov 2004; 12: 91-5.

27. Neto CD, Schimidt G, Candotti CT, Loss JF, Zaro MA, Cervieri A, Guimarães ACS. Desenvolvimento de uma plataforma de força em pedal de ciclismo. Rev Bras Biomec 2001; 2: 39-44.

28. Desiprés M. An electromyographic study of competitive road cycling conditions simulated on a treadmill. In: Nelson RC, Morehouse C (ed). Biomechanics IV. Baltimore: Universal Park Press 1974; 349-55.

29. Clarys JP, Cabri J, Gregor RJ. The muscle activity paradox during circular rhythmic leg movements. J Sports Sci 1988; 6: 229-37.

30. Welbergen $E_{1}$ Clijsen LPVM. The influence of body position on maximal performance in cycling. Eur J Appl Physiol 1990; 61: 138-42.

31. Carmo J. Biomecânica aplicada ao ciclismo. Anais do IX Congresso Brasileiro de Biomecânica, Gramado 2001; $42-7$.

32. Hug F, Decherchi P, Marqueste T, Jammes Y. EMG versus oxygen uptake cycling exercise in trained and untrained subjects. J Electromyo Kinesiol 2003; 14: 187-95.

33. Carpes FP, Bini RR, Diefenthaeler F, Nabinger E, Mota CB, Guimarães ACS. Aplicação de força no pedal em prova de ciclismo $40 \mathrm{~km}$ contra-relógio simulada: estudo preliminar. Rev. Bras. Educ. Fís. Esp 2005; 19: 105-13.

34. Broker JP, Gregor RJ. Cycling Biomechanics. In: Burke ER (ed). High-tech Cycling. Human Kinetics 1996; 145-65.

35. Herzog W, Guimarães ACS, Anton MG, Carter-Erdman KA. Moment-length relations of rectus femoris muscles of speed skaters/cyclists and runners. Med Sci Sports Exerc 1991; 23: 1289-96.

36. Savelberg HC, Meijer K. Contribution of mono and biarticular muscles to extending knee joint moments in runners and cyclists. J Appl Physiol 2003; 94: 2241-8.

37. Gregor RJ, Broker JP, Rayan MM. The biomechanics of cycling. Exerc Sport Sci Rev 1991; 19: 127-69.

38. Groot G, Welbergen E, Clijsen L, Carijs J, Cabri J, Antonis J. Power, muscular work, and external forces in cycling. Ergonomics 1994; 37: 31-42.

39. Burke ER. Physiology of cycling. In Garrret WE \& Kirkendall DT. Exercise and Sport Science. Philadelphia: Lippincott Williams \& Wilkins 2000; 759-70.

40. Gregor RJ. Biomechanics of cycling. In: Garret WE \& Kirkendal D T. Exercise and Sport Science. Philadelphia: Lippincott Williams \& Wilkins 2000; 515-37. 